This article is published with open access at Springerlink.com

\title{
LOWER BOUNDS FOR MEASURABLE CHROMATIC NUMBERS
}

\author{
Christine Bachoc, Gabriele Nebe, \\ Fernando Mário de Oliveira Filho and Frank Vallentin
}

\begin{abstract}
The Lovász theta function provides a lower bound for the chromatic number of finite graphs based on the solution of a semidefinite program. In this paper we generalize it so that it gives a lower bound for the measurable chromatic number of distance graphs on compact metric spaces.

In particular we consider distance graphs on the unit sphere. There we transform the original infinite semidefinite program into an infinite linear program which then turns out to be an extremal question about Jacobi polynomials which we solve explicitly in the limit. As an application we derive new lower bounds for the measurable chromatic number of the Euclidean space in dimensions $10, \ldots, 24$ and we give a new proof that it grows exponentially with the dimension.
\end{abstract}

\section{Introduction}

The chromatic number of the $n$-dimensional Euclidean space is the minimum number of colors needed to color each point of $\mathbb{R}^{n}$ in such a way that points at distance 1 from each other receive different colors. It is the chromatic number of the graph with vertex set $\mathbb{R}^{n}$ and in which two vertices are adjacent if their distance is 1 . We denote it by $\chi\left(\mathbb{R}^{n}\right)$.

A famous open question is to determine the chromatic number of the plane. In this case, it is only known that $4 \leq \chi\left(\mathbb{R}^{2}\right) \leq 7$, where lower and upper bounds come from simple geometric constructions. In this form the problem was considered, e.g., by Nelson, Isbell, Erdős, and Hadwiger. For historical remarks and for the best known bounds in other dimensions we refer to Székely's survey article [Szé]. The first exponential asymptotic lower bound is due to Frankl and Wilson [FrW, Th. 3]. Currently the best known asymptotic lower bound is due to Raigorodskii $[R]$ and the best known asymptotic upper bound is due to Larman and Rogers [LR]:

$$
(1.239 \ldots+o(1))^{n} \leq \chi\left(\mathbb{R}^{n}\right) \leq(3+o(1))^{n} .
$$

Keywords and phrases: Nelson-Hadwiger problem, measurable chromatic number, semidefinite programming, orthogonal polynomials, spherical codes

2000 Mathematics Subject Classification: 52C10, 52C17, 90C22

The third author was partially supported by CAPES/Brazil under grant BEX 2421/04-6. The fourth author was partially supported by the Deutsche Forschungsgemeinschaft (DFG) under grant SCHU 1503/4. 
In this paper we study a variant of the chromatic number of $\mathbb{R}^{n}$, namely the measurable chromatic number. The measurable chromatic number of $\mathbb{R}^{n}$ is the smallest number $m$ such that $\mathbb{R}^{n}$ can be partitioned into $m$ Lebesgue measurable stable sets. Here we call a set $C \subseteq \mathbb{R}^{n}$ stable if no two points in $C$ lie at distance 1 from each other. In other words, we impose that the sets of points having the same color have to be measurable. We denote the measurable chromatic number of $\mathbb{R}^{n}$ by $\chi_{\mathrm{m}}\left(\mathbb{R}^{n}\right)$. One reason to study the measurable chromatic number is that then stronger analytic tools are available.

The study of the measurable chromatic number started with Falconer $[\mathrm{F}]$, who proved that $\chi_{\mathrm{m}}\left(\mathbb{R}^{2}\right) \geq 5$. The measurable chromatic number is at least the chromatic number, and it is amusing to notice that in case of strict inequality the construction of an optimal coloring necessarily uses the axiom of choice.

Related to the chromatic number of the Euclidean space is the chromatic number of the unit sphere $S^{n-1}=\left\{x \in \mathbb{R}^{n}: x \cdot x=1\right\}$. For $-1<t<1$, we consider the graph $G(n, t)$ whose vertices are the points of $S^{n-1}$ and in which two points are adjacent if their inner product $x \cdot y$ equals $t$. The chromatic number of $G(n, t)$ and its measurable version, denoted by $\chi(G(n, t))$ and $\chi_{\mathrm{m}}(G(n, t))$ respectively, are defined as in the Euclidean case.

The chromatic number of this graph was studied by Lovász [L2], in particular in the case when $t$ is small. He showed that

$$
\begin{array}{cl}
n \leq \chi(G(n, t)) & \text { for }-1<t<1, \\
\chi(G(n, t)) \leq n+1 & \text { for }-1<t \leq-1 / n .
\end{array}
$$

Frankl and Wilson [FrW, Th.6] showed that

$$
(1+o(1))(1.13)^{n} \leq \chi_{\mathrm{m}}(G(n, 0)) \leq 2^{n-1} .
$$

The (measurable) chromatic number of $G(n, t)$ provides a lower bound for the one of $\mathbb{R}^{n}$ : After appropriate scaling, every proper coloring of $\mathbb{R}^{n}$ intersected with the unit sphere $S^{n-1}$ gives a proper coloring of the graph $G(n, t)$, and measurability is preserved by the intersection.

In this paper we present a lower bound for the measurable chromatic number of $G(n, t)$. As an application we derive new lower bounds for the measurable chromatic number of the Euclidean space in dimensions $10, \ldots, 24$ and we give a new proof that it grows exponentially with the dimension.

The lower bound is based on a generalization of the Lovász theta function [L1], which gives an upper bound to the stability number of a finite graph. Here we aim at generalizing the theta function to distance graphs in compact metric spaces. These are graphs defined on all points of the metric space where the adjacency relation only depends on the distance.

The paper is structured as follows: In section 2 we define the stability number and the fractional measurable chromatic number and give a basic inequality involving them. Then, after reviewing Lovász' original formulation of the theta function in section 3 , we give our generalization in section 4 . Like the original theta function for finite graphs, it gives an upper bound for the stability number. Moreover, in the case of the unit sphere, it can be explicitly computed, thanks to classical results 
on spherical harmonics. The material needed for spherical harmonics is given in section 5 and an explicit formulation for the theta function of $G(n, t)$ is given in section 6 .

In section 7 we choose specific values of $t$ for which we can analytically compute the theta function of $G(n, t)$. This allows us to compute the limit of the theta function for the graph $G(n, t)$ as $t$ goes to 1 in section 8 . This gives improvements on the best-known lower bounds for $\chi_{\mathrm{m}}\left(\mathbb{R}^{n}\right)$ in several dimensions. Furthermore this gives a new proof of the fact that $\chi_{\mathrm{m}}\left(\mathbb{R}^{n}\right)$ grows exponentially with $n$. Although this is an immediate consequence of the result of Frankl and Wilson (and of Raigorodskii, and also of a result of Frankl and Rödl [FrR]) and our bound of $1.165^{n}$ is not an improvement, our result is an easy consequence of the methods we present. Moreover, we think that our proof is of interest because the methods we use here are radically different from those used before. In particular, they can be applied to other metric spaces.

In section 9 we point out how to apply our generalization to distance graphs in other compact metric spaces, endowed with the continuous action of a compact group. Finally in section 10 we conclude by showing the relation between our generalization of the theta function and the theta function for finite graphs of $G(n, t)$ and by showing the relation between our generalization and the linear programming bound for spherical codes established by Delsarte, Goethals, and Seidel [DGS].

\section{The Fractional Chromatic Number and the Stability Number}

Let $G=(V, E)$ be a finite or infinite graph whose vertex set is equipped with the measure $\mu$. We assume that the measure of $V$ is finite. In this section we define the stability number and the measurable fractional chromatic number of $G$ and derive the basic inequality between these two invariants. In the case of a finite graph one recovers the classical notions if one uses the uniform measure $\mu(C)=|C|$ for $C \subseteq V$.

Let $L^{2}(V)$ be the Hilbert space of real-valued square-integrable functions defined over $V$ with inner product

$$
(f, g)=\int_{V} f(x) g(x) d \mu(x)
$$

for $f, g \in L^{2}(V)$. The constant function 1 is measurable and its squared norm is the number $(1,1)=\mu(V)$. The characteristic function of a subset $C$ of $V$ we denote by $\chi^{C}: V \rightarrow\{0,1\}$.

A subset $C$ of $V$ is called a measurable stable set if $C$ is a measurable set and if no two vertices in $C$ are adjacent. The stability number of $G$ is

$$
\alpha(G)=\sup \{\mu(C): C \subseteq V \text { is a measurable stable set }\} .
$$

Similar measure-theoretical notions of the stability number have been considered before by other authors for the case in which $V$ is the Euclidean space $\mathbb{R}^{n}$ or the sphere $S^{n-1}$. We refer the reader to the survey paper of Székely [Szé] for more information and further references.

The fractional measurable chromatic number of $G$ is denoted by $\chi_{\mathrm{m}}^{*}(G)$. It is the infimum of $\lambda_{1}+\cdots+\lambda_{k}$ where $k \geq 0$ and $\lambda_{1}, \ldots, \lambda_{k}$ are non-negative real numbers 
such that there exist measurable stable sets $C_{1}, \ldots, C_{k}$ satisfying

$$
\lambda_{1} \chi^{C_{1}}+\cdots+\lambda_{k} \chi^{C_{k}}=1 \text {. }
$$

Note that the measurable fractional chromatic number of the graph $G$ is a lower bound for its measurable chromatic number.

Proposition 2.1. We have the following basic inequality between the stability number and the measurable fractional chromatic number of a graph $G=(V, E)$ :

$$
\alpha(G) \chi_{\mathrm{m}}^{*}(G) \geq \mu(V) .
$$

So, any upper bound for $\alpha(G)$ provides a lower bound for $\chi_{\mathrm{m}}^{*}(G)$.

Proof. Let $\lambda_{1}, \ldots, \lambda_{k}$ be non-negative real numbers and $C_{1}, \ldots, C_{k}$ be measurable stable sets such that $\lambda_{1} \chi^{C_{1}}+\cdots+\lambda_{k} \chi^{C_{k}}=1$. Since $C_{i}$ is measurable, its characteristic function $\chi^{C_{i}}$ lies in $L^{2}(V)$. Hence

$$
\begin{aligned}
\left(\lambda_{1}+\cdots+\lambda_{k}\right) \alpha(G) & \geq \lambda_{1} \mu\left(C_{1}\right)+\cdots+\lambda_{k} \mu\left(C_{k}\right) \\
& =\lambda_{1}\left(\chi^{C_{1}}, 1\right)+\cdots+\lambda_{k}\left(\chi^{C_{k}}, 1\right) \\
& =(1,1) \\
& =\mu(V) .
\end{aligned}
$$

\section{The Lovász Theta Function for Finite Graphs}

In the celebrated paper [L1] Lovász introduced the theta function for finite graphs. It is an upper bound for the stability number which one can efficiently compute using semidefinite programming. In this section we review its definition and properties, which we generalize in section 4 .

The theta function of a graph $G=(V, E)$ is defined by

$$
\begin{aligned}
\vartheta(G)=\max \left\{\sum_{x \in V} \sum_{y \in V} K(x, y): K \in \mathbb{R}^{V \times V}\right. \text { is positive semidefinite, } \\
\left.\qquad \sum_{x \in V} K(x, x)=1, K(x, y)=0 \text { if }\{x, y\} \in E\right\} .
\end{aligned}
$$

Theorem 3.1. For any finite graph $G, \vartheta(G) \geq \alpha(G)$.

Although this result follows from [L1, Lem.3] and [L1, Th.4], we give a proof here to stress the analogy between the finite case and the more general case we consider in our generalization Theorem 4.1.

Proof of Theorem 3.1. Let $C \subseteq V$ be a stable set. Consider the characteristic function $\chi^{C}: V \rightarrow\{0,1\}$ of $C$ and define the matrix $K \in \mathbb{R}^{V \times V}$ by

$$
K(x, y)=\frac{1}{|C|} \chi^{C}(x) \chi^{C}(y) .
$$

Notice $K$ satisfies the conditions in (2). Moreover, we have $\sum_{x \in V} \sum_{y \in V} K(x, y)=|C|$, and so $\vartheta(G) \geq|C|$.

REMARK 3.2. There are many equivalent definitions of the theta function. Possible alternatives are reviewed by Knuth in $[\mathrm{Kn}]$. We use the one of [L1, Th.4]. 
If the graph $G$ has a nontrivial automorphism group, it is not difficult to see that one can restrict oneself in (2) to the functions $K$ which are invariant under the action of any subgroup $\Gamma$ of $\operatorname{Aut}(G)$, where $\operatorname{Aut}(G)$ is the automorphism group of $G$, i.e. it is the group of all permutations of $V$ that preserve adjacency. Here we say that $K$ is invariant under $\Gamma$ if $K(\gamma x, \gamma y)=K(x, y)$ holds for all $\gamma \in \Gamma$ and all $x, y \in V$. If moreover $\Gamma$ acts transitively on $G$, the second condition $\sum_{x \in V} K(x, x)=1$ is equivalent to $K(x, x)=1 /|V|$ for all $x \in V$.

\section{A Generalization of the Lovász Theta Function for Distance Graphs on Compact Metric Spaces}

We assume that $V$ is a compact metric space with distance function $d$. We moreover assume that $V$ is equipped with a non-negative, Borel regular measure $\mu$ for which $\mu(V)$ is finite. Let $D$ be a closed subset of the image of $d$. We define the graph $G(V, D)$ to be the graph with vertex set $V$ and edge set $E=\{\{x, y\}: d(x, y) \in D\}$.

The elements of the space $\mathcal{C}(V \times V)$ consisting of all continuous functions $K$ : $V \times V \rightarrow \mathbb{R}$ are called continuous Hilbert-Schmidt kernels; or kernels for short. In the following we only consider symmetric kernels, i.e. kernels $K$ with $K(x, y)=K(y, x)$ for all $x, y \in V$. A kernel $K \in \mathcal{C}(V \times V)$ is called positive if, for any non-negative integer $m$, any points $x_{1}, \ldots, x_{m} \in V$, and any real numbers $u_{1}, \ldots, u_{m}$, we have

$$
\sum_{i=1}^{m} \sum_{j=1}^{m} K\left(x_{i}, x_{j}\right) u_{i} u_{j} \geq 0 \text {. }
$$

We are now ready to extend the definition (2) of the Lovász theta function to the graph $G(V, D)$. We define

$$
\begin{array}{r}
\vartheta(G(V, D))=\sup \left\{\int_{V} \int_{V} K(x, y) d \mu(x) d \mu(y): K \in \mathcal{C}(V \times V)\right. \text { is positive, } \\
\left.\int_{V} K(x, x) d \mu(x)=1, K(x, y)=0 \text { if } d(x, y) \in D\right\} .
\end{array}
$$

Theorem 4.1. The theta function is an upper bound for the stability number, i.e.

$$
\vartheta(G(V, D)) \geq \alpha(G(V, D)) \text {. }
$$

Proof. Fix $\varepsilon>0$ arbitrarily. Let $C \subseteq V$ be a stable set such that $\mu(C) \geq$ $\alpha(G(V, D))-\varepsilon$. Since $\mu$ is regular, we may assume that $C$ is closed, as otherwise we could find a stable set with measure closer to $\alpha(G(V, D))$ and use a suitable inner-approximation of it by a closed set.

Note that, since $C$ is compact and stable, there must exist a number $\beta>0$ such that $|d(x, y)-\delta|>\beta$ for all $x, y \in C$ and $\delta \in D$. But then, for small enough $\xi>0$, the set

$$
B(C, \xi)=\{x \in V: d(x, C)<\xi\},
$$

where $d(x, C)$ is the distance from $x$ to the closed set $C$, is stable. Moreover, notice that $B(C, \xi)$ is open and that, since it is stable, $\mu(B(C, \xi)) \leq \alpha(G(V, D))$.

Now, the function $f: V \rightarrow[0,1]$ given by

$$
f(x)=\xi^{-1} \cdot \max \{\xi-d(x, C), 0\}
$$


for all $x \in V$ is continuous and such that $f(C)=1$ and $f(V \backslash B(C, \xi))=0$. So the kernel $K$ given by

for all $x, y \in V$ is feasible in (3).

$$
K(x, y)=\frac{1}{(f, f)} f(x) f(y)
$$

Let us estimate the objective value of $K$. Since we have

$$
(f, f) \leq \mu(B(C, \xi)) \leq \alpha(G(V, D))
$$

and

we finally have

$$
\int_{V} \int_{V} f(x) f(y) d \mu(x) d \mu(y) \geq \mu(C)^{2} \geq(\alpha(G(V, D))-\varepsilon)^{2},
$$

$$
\int_{V} \int_{V} K(x, y) d \mu(x) d \mu(y) \geq \frac{(\alpha(G(V, D))-\varepsilon)^{2}}{\alpha(G(V, D))}
$$

and, since $\varepsilon$ is arbitrary, the theorem follows.

Let us now assume that a compact group $\Gamma$ acts continuously on $V$, preserving the distance $d$. Then, if $K$ is a feasible solution for $(3)$, so is $(x, y) \mapsto K(\gamma x, \gamma y)$ for all $\gamma \in \Gamma$. Averaging on $\Gamma$ leads to a $\Gamma$-invariant feasible solution

$$
\bar{K}(x, y)=\int_{\Gamma} K(\gamma x, \gamma y) d \gamma,
$$

where $d \gamma$ denotes the Haar measure on $\Gamma$ normalized so that $\Gamma$ has volume 1 . Moreover, observe that the objective value of $\bar{K}$ is the same as that of $K$. Hence we can restrict ourselves in (3) to $\Gamma$-invariant kernels. If moreover $V$ is homogeneous under the action of $\Gamma$, the second condition in (3) may be replaced by $K(x, x)=1 / \mu(V)$ for all $x \in V$.

We are mostly interested in the case in which $V$ is the unit sphere $S^{n-1}$ endowed with the Euclidean metric of $\mathbb{R}^{n}$, and in which $D$ is a singleton. If $D=\{\delta\}$ and $\delta^{2}=2-2 t$, so that $d(x, y)=\delta$ if and only if $x \cdot y=t$, the graph $G\left(S^{n-1}, D\right)$ is denoted by $G(n, t)$. Since the unit sphere is homogeneous under the action of the orthogonal group $\mathrm{O}\left(\mathbb{R}^{n}\right)$, the previous remarks apply.

\section{Harmonic Analysis on the Unit Sphere}

It turns out that the continuous positive Hilbert-Schmidt kernels on the sphere have a nice description coming from classical results of harmonic analysis reviewed in this section. This allows for the calculation of $\vartheta(G(n, t))$. For information on spherical harmonics we refer to [AAR, Ch. 9] and [VK].

The unit sphere $S^{n-1}$ is homogeneous under the action of the orthogonal group $\mathrm{O}\left(\mathbb{R}^{n}\right)=\left\{A \in \mathbb{R}^{n \times n}: A^{t} A=I_{n}\right\}$, where $I_{n}$ denotes the identity matrix. Moreover, it is two-point homogeneous, meaning that the orbits of $\mathrm{O}\left(\mathbb{R}^{n}\right)$ on pairs of points are characterized by the value of their inner product. The orthogonal group acts on $L^{2}\left(S^{n-1}\right)$ by $A f(x)=f\left(A^{-1} x\right)$, and $L^{2}\left(S^{n-1}\right)$ is equipped with the standard $\mathrm{O}\left(\mathbb{R}^{n}\right)$-invariant inner product

$$
(f, g)=\int_{S^{n-1}} f(x) g(x) d \omega(x)
$$


for the standard surface measure $\omega$. The surface area of the unit sphere is $\omega_{n}=$ $(1,1)=2 \pi^{n / 2} / \Gamma(n / 2)$.

It is a well-known fact (see, e.g. [VK, Ch. 9.2]) that the Hilbert space $L^{2}\left(S^{n-1}\right)$ decomposes under the action of $\mathrm{O}\left(\mathbb{R}^{n}\right)$ into orthogonal subspaces

$$
L^{2}\left(S^{n-1}\right)=H_{0} \perp H_{1} \perp H_{2} \perp \ldots,
$$

where $H_{k}$ is isomorphic to the $\mathrm{O}\left(\mathbb{R}^{n}\right)$-irreducible space

$$
\operatorname{Harm}_{k}=\left\{f \in R\left[x_{1}, \ldots, x_{n}\right]: f \text { homogeneous, } \operatorname{deg} f=k, \sum_{i=1}^{n} \frac{\partial^{2}}{\partial x_{i}^{2}} f=0\right\}
$$

of harmonic polynomials in $n$ variables which are homogeneous and have degree $k$. We set $h_{k}=\operatorname{dim}\left(\operatorname{Harm}_{k}\right)=\left(\begin{array}{c}n+k-1 \\ n-1\end{array}\right)-\left(\begin{array}{c}n+k-3 \\ n-1\end{array}\right)$. The equality in (5) means that every $f \in L^{2}\left(S^{n-1}\right)$ can be uniquely written in the form $f=\sum_{k=0}^{\infty} p_{k}$, where $p_{k} \in H_{k}$, and where the convergence is in the $L^{2}$-norm.

The addition formula (see e.g. [AAR, Ch. 9.6]) plays a central role in the characterization of $\mathrm{O}\left(\mathbb{R}^{n}\right)$-invariant kernels: For any orthonormal basis $e_{k, 1}, \ldots, e_{k, h_{k}}$ of $H_{k}$ and for any pair of points $x, y \in S^{n-1}$ we have

$$
\sum_{i=1}^{h_{k}} e_{k, i}(x) e_{k, i}(y)=\frac{h_{k}}{\omega_{n}} P_{k}^{(\alpha, \alpha)}(x \cdot y)
$$

where $P_{k}^{(\alpha, \alpha)}$ is the normalized Jacobi polynomial of degree $k$ with parameters $(\alpha, \alpha)$, with $P_{k}^{(\alpha, \alpha)}(1)=1$ and $\alpha=(n-3) / 2$. The Jacobi polynomials with parameters $(\alpha, \beta)$ are orthogonal polynomials for the weight function $(1-u)^{\alpha}(1+u)^{\beta}$ on the interval $[-1,1]$. We denote by $P_{k}^{(\alpha, \beta)}$ the normalized Jacobi polynomial of degree $k$ with normalization $P_{k}^{(\alpha, \beta)}(1)=1$.

In $[S, T h .1]$ Schoenberg gave a characterization of the continuous kernels which are positive and $\mathrm{O}\left(\mathbb{R}^{n}\right)$-invariant: They are those which lie in the cone spanned by the kernels $(x, y) \mapsto P_{k}^{(\alpha, \alpha)}(x \cdot y)$. More precisely, a continuous kernel $K \in \mathcal{C}\left(S^{n-1} \times S^{n-1}\right)$ is $\mathrm{O}\left(\mathbb{R}^{n}\right)$-invariant and positive if and only if there exist nonnegative real numbers $f_{0}, f_{1}, \ldots$ such that $K$ can be written as

$$
K(x, y)=\sum_{k=0}^{\infty} f_{k} P_{k}^{(\alpha, \alpha)}(x \cdot y)
$$

where the convergence is absolute and uniform.

\section{The Theta Function of $G(n, t)$}

We obtain from section 4 in the case $V=S^{n-1}, D=\{\sqrt{2-2 t}\}$, and $\Gamma=\mathrm{O}\left(\mathbb{R}^{n}\right)$, the following characterization of the theta function of the graph $G(n, t)=G\left(S^{n-1}, D\right)$ :

$$
\begin{aligned}
& \vartheta(G(n, t))=\max \left\{\int_{S^{n-1}} \int_{S^{n-1}} K(x, y) d \omega(x) d \omega(y):\right. \\
& K \in \mathcal{C}\left(S^{n-1} \times S^{n-1}\right) \text { is positive, } K \text { is invariant under } \mathrm{O}\left(\mathbb{R}^{n}\right), \\
& \left.K(x, x)=1 / \omega_{n} \text { for all } x \in S^{n-1}, K(x, y)=0 \text { if } x \cdot y=t\right\} .
\end{aligned}
$$

(It will be clear later that the maximum above indeed exists.) 
Corollary 6.1. We have

$$
\omega_{n} / \vartheta(G(n, t)) \leq \chi_{\mathrm{m}}^{*}(G(n, t)) .
$$

Proof. Immediate from Theorem 4.1 and the considerations in section 2 .

A result of de Bruijn and Erdős [BrE] implies that the chromatic number of $G(n, t)$ is attained by a finite subgraph of it. So one might wonder if computing the theta function for a finite subgraph of $G(n, t)$ could give a better bound than the previous corollary. This is not the case as we will show in section 10 .

The theta function for finite graphs has the important property that it can be computed in polynomial time, in the sense that it can be approximated with arbitrary precision using semidefinite programming. We now turn to the problem of computing the generalization (8).

First, we apply Schoenberg's characterization (7) of the continuous kernels which are $\mathrm{O}\left(\mathbb{R}^{n}\right)$-invariant and positive. This transforms the original formulation (3), which is a semidefinite programming problem in infinitely many variables having infinitely many constraints, into the following linear programming problem with optimization variables $f_{k}$ :

$$
\begin{aligned}
& \vartheta(G(n, t))=\max \left\{\omega_{n}^{2} f_{0}: f_{k} \geq 0 \text { for } k=0,1, \ldots,\right. \\
& \left.\qquad \sum_{k=0}^{\infty} f_{k}=1 / \omega_{n}, f_{0}+\sum_{k=1}^{\infty} f_{k} P_{k}^{(\alpha, \alpha)}(t)=0\right\},
\end{aligned}
$$

where $\alpha=(n-3) / 2$.

To obtain (9) we simplified the objective function in the following way. Because of the orthogonal decomposition (5) and because the subspace $H_{0}$ contains only the constant functions, we have

$$
\int_{S^{n-1}} \int_{S^{n-1}} \sum_{k=0}^{\infty} f_{k} P_{k}^{(\alpha, \alpha)}(x \cdot y) d \omega(x) d \omega(y)=\omega_{n}^{2} f_{0} .
$$

We furthermore used $P_{0}^{(\alpha, \alpha)}=1$ and $P_{k}^{(\alpha, \alpha)}(1)=1$.

Theorem 6.2. Let $m(t)$ be the minimum of $P_{k}^{(\alpha, \alpha)}(t)$ for $k=0,1, \ldots$ Then the optimal value of (9) is equal to

$$
\vartheta(G(n, t))=\omega_{n} \frac{m(t)}{m(t)-1} .
$$

Proof. We first claim that the minimum $m(t)$ exists and is negative. Indeed, if $P_{k}^{(\alpha, \alpha)}(t) \geq 0$ for all $k \geq 1$, then (9) either has no solution (in the case that all $P_{k}^{(\alpha, \alpha)}(t)$ are positive) or $f_{0}=0$ in any solution, which contradicts Theorem 4.1. So we know that for some $k \geq 1, P_{k}^{(\alpha, \alpha)}(t)<0$. This, combined with the fact that $P_{k}^{(\alpha, \alpha)}(t)$ goes to zero as $k$ goes to infinity (cf. [AAR, Ch.6.6] or [Sz, Ch. 8.22]), proves the claim.

Let $k^{*}$ be so that $m(t)=P_{k^{*}}^{(\alpha, \alpha)}(t)$. It is easy to see that there is an optimal solution of $(9)$ in which only $f_{0}$ and $f_{k^{*}}$ are positive. Hence, solving the resulting 
system

$$
\begin{aligned}
& f_{0}+f_{k^{*}}=1 / \omega_{n}, \\
& f_{0}+f_{k^{*}} m(t)=0,
\end{aligned}
$$

gives $f_{0}=m(t) /\left(\omega_{n}(m(t)-1)\right)$ and $f_{k^{*}}=-1 /\left(\omega_{n}(m(t)-1)\right)$ and the theorem follows.

ExAmple 6.3. The minimum of $P_{k}^{(\alpha, \alpha)}(0.9999)$ for $\alpha=(24-3) / 2$ is attained at $k=1131$. It is a rational number and its first decimal digits are -0.00059623 .

\section{Analytic Solutions}

In this section we compute the value

$$
m(t)=\min \left\{P_{k}^{(\alpha, \alpha)}(t): k=0,1, \ldots\right\}
$$

for specific values of $t$. Namely we choose $t$ to be the largest zero of an appropriate Jacobi polynomial.

Key for the discussion to follow is the interlacing property of the zeroes of orthogonal polynomials. It says (cf. [Sz, Th. 3.3.2]) that between any pair of consecutive zeroes of $P_{k}^{(\alpha, \alpha)}$ there is exactly one zero of $P_{k-1}^{(\alpha, \alpha)}$.

We denote the zeros of $P_{k}^{(\alpha, \beta)}$ by $t_{k, j}^{(\alpha, \beta)}$ with $j=1, \ldots, k$ and with the increasing ordering $t_{k, j}^{(\alpha, \beta)}<t_{k, j+1}^{(\alpha, \beta)}$. We shall need the following collection of identities:

$$
\begin{gathered}
\left(1-u^{2}\right) \frac{d^{2} P_{k}^{(\alpha, \alpha)}}{d u^{2}}-(2 \alpha+2) u \frac{d P_{k}^{(\alpha, \alpha)}}{d u}+k(k+2 \alpha+1) P_{k}^{(\alpha, \alpha)}=0, \\
(-1)^{k} P_{k}^{(\alpha, \alpha)}(-u)=P_{k}^{(\alpha, \alpha)}(u), \\
(-1)^{k}(\alpha+1) P_{k}^{(\alpha, \alpha+1)}(-u)=(k+\alpha+1) P_{k}^{(\alpha+1, \alpha)}(u), \\
(2 \alpha+2) \frac{d P_{k}^{(\alpha, \alpha)}}{d u}=k(k+2 \alpha+1) P_{k-1}^{(\alpha+1, \alpha+1)}, \\
(2 \alpha+2) P_{k}^{(\alpha, \alpha+1)}=(k+2 \alpha+2) P_{k}^{(\alpha+1, \alpha+1)}-k P_{k-1}^{(\alpha+1, \alpha+1)}, \\
(2 k+2 \alpha+2) P_{k}^{(\alpha+1, \alpha)}=(k+2 \alpha+2) P_{k}^{(\alpha+1, \alpha+1)}+k P_{k-1}^{(\alpha+1, \alpha+1)}, \\
(k+\alpha+1) P_{k}^{(\alpha+1, \alpha)}=(\alpha+1) \frac{P_{k}^{(\alpha, \alpha)}-P_{k+1}^{(\alpha, \alpha)}}{1-u} .
\end{gathered}
$$

They can all be found in [AAR, Ch.6], although with different normalization. Formula (10) is [AAR, (6.3.9)]; (11) and (12) are [AAR, (6.4.23)]; (13) is [AAR, (6.3.8)], (14) is [AAR, (6.4.21)]; (15) follows by the change of variables $u \mapsto-u$ from (14) and (11), (12); (16) is [AAR, (6.4.20)].

Proposition 7.1. Let $t=t_{k-1, k-1}^{(\alpha+1, \alpha+1)}$ be the largest zero of the Jacobi polynomial $P_{k-1}^{(\alpha+1, \alpha+1)}$. Then, $m(t)=P_{k}^{(\alpha, \alpha)}(t)$.

Proof. We start with the following crucial observation: From (13), $t$ is a zero of the derivative of $P_{k}^{(\alpha, \alpha)}$. Hence it is a minimum of $P_{k}^{(\alpha, \alpha)}$ because it is the last extremal 
value in the interval $[-1,1]$ and because $P_{k}^{(\alpha+1, \alpha+1)}(1)=1$, whence (using (13)) $P_{k}^{(\alpha, \alpha)}(u)$ is increasing on $[t, 1]$.

Now we prove that $P_{k}^{(\alpha, \alpha)}(t)<P_{j}^{(\alpha, \alpha)}(t)$ for all $j \neq k$ where we treat the cases $j<k$ and $j>k$ separately.

It turns out that the sequence $P_{j}^{(\alpha, \alpha)}(t)$ is decreasing for $j \leq k$. From (16), the sign of $P_{j}^{(\alpha, \alpha)}(t)-P_{j+1}^{(\alpha, \alpha)}(t)$ equals the sign of $P_{j}^{(\alpha+1, \alpha)}(t)$. We have the inequalities $t_{j, j}^{(\alpha+1, \alpha)} \leq t_{k-1, k-1}^{(\alpha+1, \alpha)}<t_{k-1, k-1}^{(\alpha+1, \alpha+1)}=t$.

The first one is a consequence of the interlacing property. From (15) one can deduce that $P_{k-1}^{(\alpha+1, \alpha)}$ has exactly one zero in the interval $\left[t_{k-2, i-1}^{(\alpha+1, \alpha+1)}, t_{k-1, i}^{(\alpha+1, \alpha+1)}\right]$ since it changes sign at the extreme points of it, and by the same argument $P_{k-1}^{(\alpha+1, \alpha)}$ has a zero left to $t_{k-1,1}^{(\alpha+1, \alpha+1)}$. Thus, $t_{k-1, k-1}^{(\alpha+1, \alpha)}<t_{k-1, k-1}^{(\alpha+1, \alpha+1)}=t$. So $t$ lies to the right of the largest zero of $P_{j}^{(\alpha+1, \alpha)}$ and hence $P_{j}^{(\alpha+1, \alpha)}(t)>0$ which shows that $P_{j}^{(\alpha, \alpha)}(t)-P_{j+1}^{(\alpha, \alpha)}(t)>0$ for $j<k$.

Let us consider the case $j>k$. The inequality [AAR, (6.4.19)] implies that

$$
\text { for all } j>k, \quad P_{k}^{(\alpha, \alpha)}\left(t_{k-1, k-1}^{(\alpha+1, \alpha+1)}\right)<P_{j}^{(\alpha, \alpha)}\left(t_{j-1, j-1}^{(\alpha+1, \alpha+1)}\right) \text {. }
$$

The next observation, which finishes the proof of the lemma, is stated in [AAR, (6.4.24)] only for the case $\alpha=0$ :

$$
\text { for all } j \geq 2, \quad \min \left\{P_{j}^{(\alpha, \alpha)}(u): u \in[0,1]\right\}=P_{j}^{(\alpha, \alpha)}\left(t_{j-1, j-1}^{(\alpha+1, \alpha+1)}\right) .
$$

To prove it consider

$$
g(u)=P_{j}^{(\alpha, \alpha)}(u)^{2}+\frac{1-u^{2}}{j(j+2 \alpha+1)}\left(\frac{d P_{j}^{(\alpha, \alpha)}}{d u}\right)^{2} .
$$

Applying (10) in the computation of $g^{\prime}$ shows that

$$
g^{\prime}(u)=\frac{(4 \alpha+2) u}{j(j+2 \alpha+1)}\left(\frac{d P_{j}^{(\alpha, \alpha)}}{d u}\right)^{2} .
$$

The polynomial $g^{\prime}$ takes positive values on $[0,1]$ and hence $g$ is increasing on this interval. In particular,

$$
g\left(t_{j-1, i-1}^{(\alpha+1, \alpha+1)}\right)<g\left(t_{j-1, i}^{(\alpha+1, \alpha+1)}\right) \quad \text { for all } i \leq j-1 \text { with } t_{j-1, i-1}^{(\alpha+1, \alpha+1)} \geq 0,
$$

which simplifies to

$$
P_{j}^{(\alpha, \alpha)}\left(t_{j-1, i-1}^{(\alpha+1, \alpha+1)}\right)^{2}<P_{j}^{(\alpha, \alpha)}\left(t_{j-1, i}^{(\alpha+1, \alpha+1)}\right)^{2} .
$$

Since $t_{j-1, i}^{(\alpha+1, \alpha+1)}$ are the local extrema of $P_{j}^{(\alpha, \alpha)}$, we have proved (18).

\section{New Lower Bounds for the Euclidean Space}

In this section we give new lower bounds for the measurable chromatic number of the Euclidean space for dimensions $10, \ldots, 24$. This improves on the previous best known lower bounds due to Székely and Wormald [SzéW]. Table 8.1 compares the values. Furthermore we give a new proof that the measurable chromatic number grows exponentially with the dimension. 
For this we give a closed expression for $\lim _{t \rightarrow 1} m(t)$ which involves the Bessel function $J_{\alpha}$ of the first kind of order $\alpha=(n-3) / 2$ (see e.g. [AAR, Ch. 4]). The appearance of Bessel functions here is due to the fact that the largest zero of the Jacobi polynomial $P_{k}^{(\alpha, \alpha)}$ behaves like the first positive zero $j_{\alpha}$ of the Bessel function $J_{\alpha}$. More precisely, it is known [AAR, Th. 4.14.1] that, for the largest zero $t_{k, k}^{(\alpha+1, \beta)}=\cos \theta_{k}$ of the polynomial $P_{k}^{(\alpha+1, \beta)}$,

$$
\lim _{k \rightarrow \infty} k \theta_{k}=j_{\alpha+1}
$$

and, with our normalization (cf. [AAR, Th.4.11.6]),

$$
\lim _{k \rightarrow \infty} P_{k}^{(\alpha, \alpha)}\left(\cos \frac{u}{k}\right)=2^{\alpha} \Gamma(\alpha+1) \frac{J_{\alpha}(u)}{u^{\alpha}} .
$$

Theorem 8.1. We have

$$
\lim _{t \rightarrow 1} m(t)=2^{\alpha} \Gamma(\alpha+1) \frac{J_{\alpha}\left(j_{\alpha+1}\right)}{\left(j_{\alpha+1}\right)^{\alpha}} .
$$

Proof. First we show that

$$
\lim _{k \rightarrow \infty} P_{k}^{(\alpha, \alpha)}\left(t_{k-1, k-1}^{(\alpha+1, \beta)}\right)=2^{\alpha} \Gamma(\alpha+1) \frac{J_{\alpha}\left(j_{\alpha+1}\right)}{\left(j_{\alpha+1}\right)^{\alpha}} .
$$

We estimate the difference

that we upper bound by

$$
\left|P_{k}^{(\alpha, \alpha)}\left(t_{k-1, k-1}^{(\alpha+1, \beta)}\right)-2^{\alpha} \Gamma(\alpha+1) \frac{J_{\alpha}\left(j_{\alpha+1}\right)}{\left(j_{\alpha+1}\right)^{\alpha}}\right|,
$$

$$
\begin{aligned}
& \left|P_{k}^{(\alpha, \alpha)}\left(t_{k-1, k-1}^{(\alpha+1, \beta)}\right)-P_{k}^{(\alpha, \alpha)}\left(\cos \frac{j_{\alpha+1}}{k}\right)\right| \\
& \quad+\left|P_{k}^{(\alpha, \alpha)}\left(\cos \frac{j_{\alpha+1}}{k}\right)-2^{\alpha} \Gamma(\alpha+1) \frac{J_{\alpha}\left(j_{\alpha+1}\right)}{\left(j_{\alpha+1}\right)^{\alpha}}\right| .
\end{aligned}
$$

The second term tends to 0 from (20). Define $\theta_{k-1}$ by $t_{k-1, k-1}^{(\alpha+1, \beta)}=\cos \theta_{k-1}$. By the mean value theorem we have

$$
\begin{aligned}
& \left|P_{k}^{(\alpha, \alpha)}\left(t_{k-1, k-1}^{(\alpha+1, \beta)}\right)-P_{k}^{(\alpha, \alpha)}\left(\cos \frac{j_{\alpha+1}}{k}\right)\right| \\
& \leq\left(\max _{u \in[-1,1]}\left|\frac{d P_{k}^{(\alpha, \alpha)}}{d u}\right|\right)\left|\cos \theta_{k-1}-\cos \frac{j_{\alpha+1}}{k}\right| \\
& \leq\left(\max _{u \in[-1,1]}\left|\frac{d P_{k}^{(\alpha, \alpha)}}{d u}\right|\right)\left(\max _{\theta \in I_{k}}|\sin \theta|\right)\left|\theta_{k-1}-\frac{j_{\alpha+1}}{k}\right|,
\end{aligned}
$$

where $I_{k}$ denotes the interval with extremes $\theta_{k-1}$ and $\frac{j_{\alpha+1}}{k}$. Then, with (19),

and for all $\theta \in I_{k}$

$$
\begin{aligned}
\theta_{k-1}-\frac{j_{\alpha+1}}{k} & =\theta_{k-1}-\frac{j_{\alpha+1}}{k-1}+\frac{j_{\alpha+1}}{k(k-1)} \\
& =\frac{1}{k-1}\left((k-1) \theta_{k-1}-j_{\alpha+1}\right)+\frac{j_{\alpha+1}}{k(k-1)}=o\left(\frac{1}{k}\right),
\end{aligned}
$$

$$
|\sin \theta| \leq|\theta| \leq \frac{j_{\alpha+1}}{k}+\left|\theta_{k-1}-\frac{j_{\alpha+1}}{k}\right|=O\left(\frac{1}{k}\right) .
$$


From (13),

Hence we have proved that

$$
\max _{u \in[-1,1]}\left|\frac{d P_{k}^{(\alpha, \alpha)}}{d u}\right| \sim k^{2}
$$

$$
\lim _{k \rightarrow \infty}\left|P_{k}^{(\alpha, \alpha)}\left(t_{k-1, k-1}^{(\alpha+1, \beta)}\right)-P_{k}^{(\alpha, \alpha)}\left(\cos \frac{j_{\alpha+1}}{k}\right)\right|=0,
$$

and (21) follows.

Since the zeros $t_{k, k}^{(\alpha, \beta)}$ tend to 1 as $k$ tends to infinity, to prove the theorem it suffices to show that $\lim _{t \rightarrow 1} m(t)$ exists. This follows from $(21)$ and the following two facts which hold for all $k \geq 2$ :

and

$$
P_{k}^{(\alpha, \alpha)}\left(t_{k-1, k-1}^{(\alpha+1, \alpha+1)}\right) \leq m(t) \text { for all } t \geq t_{k-1, k-1}^{(\alpha+1, \alpha+1)}
$$

$$
m(t) \leq P_{k+1}^{(\alpha, \alpha)}\left(t_{k, k}^{(\alpha+1, \alpha)}\right) \quad \text { for all } t \in\left[t_{k-1, k-1}^{(\alpha+1, \alpha+1)}, t_{k, k}^{(\alpha+1, \alpha+1)}\right] .
$$

Fact (22) follows from (18) and [AAR, (6.4.19)]. For establishing fact (23) we argue as follows: As in the proof of Proposition 7.1, we use (15) to show that $P_{k}^{(\alpha+1, \alpha)}$ has exactly one zero in the interval $\left[t_{k-1, k-1}^{(\alpha+1, \alpha+1)}, t_{k, k}^{(\alpha+1, \alpha+1)}\right]$, namely $t_{k, k}^{(\alpha+1, \alpha)}$. From (16) we then see that $t_{k, k}^{(\alpha+1, \alpha)}$ is the only point in this interval where $P_{k}^{(\alpha, \alpha)}$ and $P_{k+1}^{(\alpha, \alpha)}$ coincide. Now it follows from the interlacing property that $P_{k}^{(\alpha, \alpha)}$ is increasing in the interval and that $P_{k+1}^{(\alpha, \alpha)}$ is decreasing in the interval, and we are done.

Corollary 8.2. We have

where $\alpha=(n-3) / 2$.

$$
\chi_{\mathrm{m}}\left(\mathbb{R}^{n}\right) \geq 1+\frac{\left(j_{\alpha+1}\right)^{\alpha}}{2^{\alpha} \Gamma(\alpha+1)\left|J_{\alpha}\left(j_{\alpha+1}\right)\right|},
$$

We use this corollary to derive new lower bounds for $n=10, \ldots, 24$. We give them in Table 8.1. For $n=2, \ldots, 8$, our bounds are worse than the existing ones and for $n=9$ our bound is 35 which is also the best-known one.

In fact Oliveira and Vallentin $[\mathrm{OV}]$ show, by different methods, that the above bound is actually a bound for $\chi_{\mathrm{m}}\left(\mathbb{R}^{n-1}\right)$. This then gives improved bounds starting from $n=4$. With the use of additional geometric arguments one can also get a new bound for $n=3$ in this framework.

We can also use the corollary to show that our bound is exponential in the dimension. To do so we use the inequalities (cf. [AAR, (4.14.1)] and [W, §15.3, p. 485])

$$
j_{\alpha+1}>j_{\alpha}>\alpha
$$

and (cf. $[$ AAR, (4.9.13)])

$$
\left|J_{\alpha}(x)\right| \leq 1 / \sqrt{2}
$$

to obtain

$$
\frac{\left(j_{\alpha+1}\right)^{\alpha}}{2^{\alpha} \Gamma(\alpha+1)\left|J_{\alpha}\left(j_{\alpha+1}\right)\right|}>\sqrt{2} \frac{\alpha^{\alpha}}{2^{\alpha} \Gamma(\alpha+1)},
$$

and with Stirling's formula $\Gamma(\alpha+1) \sim \alpha^{\alpha} e^{-\alpha} \sqrt{2 \pi \alpha}$ we have that the exponential term is $(e / 2)^{\alpha} \sim(1.165)^{n}$. 


\begin{tabular}{c|c|c}
$n$ & $\begin{array}{c}\text { best lower bound } \\
\text { previously known for } \chi_{\mathrm{m}}\left(\mathbb{R}^{n}\right)\end{array}$ & $\begin{array}{c}\text { new lower bound } \\
\text { for } \chi_{\mathrm{m}}\left(\mathbb{R}^{n}\right)\end{array}$ \\
\hline 10 & 45 & 48 \\
11 & 56 & 64 \\
12 & 70 & 85 \\
13 & 84 & 113 \\
14 & 102 & 147 \\
15 & 119 & 191 \\
16 & 148 & 248 \\
17 & 174 & 319 \\
18 & 194 & 408 \\
19 & 263 & 521 \\
20 & 315 & 662 \\
21 & 374 & 839 \\
22 & 526 & 1060 \\
23 & 754 & 1336 \\
24 & 933 & 1679
\end{tabular}

Table 8.1: Lower bounds for $\chi_{\mathrm{m}}\left(\mathbb{R}^{n}\right)$.

\section{Other Spaces}

In this section we want to go back to our generalization (3) of the theta function and discuss its computation in more general situations than the one of the graph $G(n, t)$ encountered in section 6 . We assume that a compact group $\Gamma$ acts continuously on $V$. Then, the computation only depends on the orthogonal decomposition of the space of $L^{2}$-functions (24).

9.1 Two-point homogeneous spaces. First, it is worth noticing that all results in section 6 are valid - one only has to use the appropriate zonal polynomials and appropriate volumes - for distance graphs in infinite, two-point homogeneous, compact metric spaces where edges are given by exactly one distance.

If one considers distance graphs in infinite, compact, two-point homogeneous metric spaces with $s$ distances, then it is helpful to consider a dual formulation of (9). It is an infinite linear programming problem in dimension $s+1$ which in the case of the unit sphere has the following form:

$$
\begin{aligned}
\min \left\{z_{1} / \omega_{n}: z_{1}+z_{t_{1}}+\cdots+z_{t_{s}} \geq \omega_{n}^{2},\right. & \\
& \left.z_{1}+z_{t_{1}} P_{k}^{(\alpha, \alpha)}\left(t_{1}\right)+\cdots+z_{t_{s}} P_{k}^{(\alpha, \alpha)}\left(t_{s}\right) \geq 0 \text { for } k=1,2, \ldots\right\},
\end{aligned}
$$

where $t_{1}, \ldots, t_{s}$ are the inner products defining the edges of our graph.

9.2 Symmetric spaces. Next we may consider infinite compact metric spaces $V$ which are not two-point homogeneous but symmetric. Since the space $L^{2}(V)$ still has a multiplicity-free orthogonal decomposition one gets a linear programming bound, but with the additional complication that one has to work with multivariate zonal 
polynomials. The most prominent case of the Grassmann manifold was considered by the first author in [B] in the context of finding upper bounds for finite codes.

9.3 General homogeneous spaces. For the most general case one would have multiplicities $m_{k}$ in the decomposition of $L^{2}(V)$ which is given by the Peter-Weyl theorem:

$$
L^{2}(V)=\left(H_{0,1} \perp \ldots \perp H_{0, m_{0}}\right) \perp\left(H_{1,1} \perp \ldots \perp H_{1, m_{1}}\right) \perp \ldots,
$$

where $H_{k, l}$ are $\Gamma$-irreducible subspaces which are equivalent whenever their first index coincides. In this case one uses Bochner's characterization of the continuous, $\Gamma$-invariant, positive kernels given in $[\mathrm{Bo}, \S \mathrm{III}]$ which yields a true semidefinite programming problem for the computation of $\vartheta$.

\section{Second Generalization}

In this section we first show how our generalization relates to the theta function of finite subgraphs of $G(n, t)$. We prove that computing the theta function for any finite subgraph of $G(n, t)$ does not give a better bound than the one of Corollary 6.1. For this we introduce a second generalization of the theta function. Then we show how our second generalization relates to the linear programming bound of Delsarte.

10.1 Finite subgraphs. To compute a bound for the measurable chromatic number of the graph $G(n, t)$ we compute $\vartheta(G(n, t))$, which is an upper bound for $\alpha(G(n, t))$, and then $\omega_{n} / \vartheta(G(n, t))$ is a lower bound for $\chi_{\mathrm{m}}(G(n, t))$.

When $G=(V, E)$ is a finite graph, this approach corresponds to computing $\vartheta(G)$ and using $|V| / \vartheta(G)$ as a lower bound for $\chi(G)$. However, this is in general not the best bound we can obtain for $\chi(G)$ from the theta function. Indeed, for a finite graph $G$, the so-called sandwich theorem says that

$$
\alpha(G) \leq \vartheta(G) \leq \chi(\bar{G})
$$

(Theorem 3.1 only gives the first inequality, Lovász [L1, Proof of Cor. 3] gives the second), where $\bar{G}$ is the complement of $G$, the graph with the same vertex set as $G$ and in which two vertices are adjacent if and only if they are nonadjacent in $G$.

Moreover, for a finite graph $G=(V, E)$, we have

$$
\vartheta(G) \vartheta(\bar{G}) \geq|V|
$$

(cf. [L1, Cor. 2]). For some graphs (e.g. stars), this inequality is strict, hence in these cases $\vartheta(\bar{G})$ would provide us with a better lower bound for $\chi(G)$ than $|V| / \vartheta(G)$ would. But when $V$ is homogeneous we actually have equality in (25) (cf. [L1, Th. 8]). In this case, both bounds for $\chi(G)$ coincide.

Something similar happens for our infinite distance graph $G(n, t)$. The complement of $G(n, t)$ is the graph in which any two distinct points on the unit sphere whose inner product is not $t$ are adjacent. We cannot use our generalization of the theta function to define $\vartheta(\overline{G(n, t)})$. However, we may use a different (and for finite graphs, equivalent) definition of $\vartheta$ (cf. [L1, Th.3]), which for a finite graph $G=(V, E)$ is

$$
\vartheta(\bar{G})=\min \left\{\lambda: K \in \mathbb{R}^{V \times V}\right. \text { is positive semidefinite, }
$$




$$
\begin{aligned}
& K(x, x)=\lambda-1 \text { for all } x \in V, \\
& K(x, y)=-1 \text { if }\{x, y\} \in E\} .
\end{aligned}
$$

The generalization of this definition, applied to $\overline{G(n, t)}$ and with the symmetry taken into account, is described below. We choose to write $\bar{\vartheta}(G(n, t))$ instead of $\vartheta(\overline{G(n, t)})$ to emphasize that the two ways to define the theta function are not equivalent for our infinite graph. So we have

$$
\begin{aligned}
\bar{\vartheta}(G(n, t))=\min \{\lambda: & K \in \mathcal{C}\left(S^{n-1} \times S^{n-1}\right) \text { is positive, } \\
& K \text { is invariant under } \mathrm{O}\left(\mathbb{R}^{n}\right), \\
& K(x, x)=\lambda-1 \text { for all } x \in S^{n-1}, \\
& K(x, y)=-1 \text { if } x \cdot y=t\} .
\end{aligned}
$$

By decomposing the kernel $K$ with the help of the Jacobi polynomials as done in section 6 , we may compute the optimal value of the optimization problem (27), and in doing so we find out that

$$
\vartheta(G(n, t)) \bar{\vartheta}(G(n, t))=\omega_{n}
$$

so that we have the analogue of $\vartheta(G) \vartheta(\bar{G})=|V|$ for our infinite distance graph on the unit sphere.

This also provides us with the connection to the theta function of finite subgraphs of $G(n, t)$ claimed in section 6 . If $H=(V, E)$ is a finite subgraph of $G(n, t)$, then $\vartheta(\bar{H})$ provides a lower bound for $\chi(H)$, which in turn is a lower bound for $\chi_{\mathrm{m}}(G(n, t))$. It could be that for some finite subgraph $H$ of $G(n, t)$ this lower bound would be better than the one provided by $\vartheta(G(n, t))$. This is, however, not the case. Indeed, if $K$ is an optimal solution for (27), the restriction of $K$ to $V \times V$ is a feasible solution to the optimization problem (26) defining $\vartheta(\bar{H})$, hence $\vartheta(\bar{H}) \leq \bar{\vartheta}(G(n, t))$, which is our bound for $\chi_{\mathrm{m}}(G(n, t))$.

10.2 Delsarte's linear programming bound. The second generalization $\bar{\vartheta}$ of the theta function is closely related to the linear programming bound for finite codes established by Delsarte in $[\mathrm{D}]$ and put into the framework of group representations, which we use here, by Kabatiansky and Levenshtein in [KL]. Here we devise an explicit connection between these two bounds. The connection between the linear programming bound and the theta function was already observed by McEliece, Rodemich, Rumsey Jr. in [MRR] and independently by Schrijver in [Sc] in the case of finite graphs.

Consider the graph on the unit sphere where two distinct points are adjacent whenever their inner product lies in the open interval $[-1, t]$. We denote this graph by $G(n,[-1, t])$. Stable sets in the complement of this graph are finite and consist of points on the unit sphere with minimal angular distance $\arccos t$.

Now the second generalization (26) applied to $G(n,[-1, t])$ is

$$
\begin{aligned}
\bar{\vartheta}(G(n,[-1, t]))=\inf \{\lambda: & K \in \mathcal{C}\left(S^{n-1} \times S^{n-1}\right) \text { is positive, } \\
& K \text { is invariant under } \mathrm{O}\left(\mathbb{R}^{n}\right), \\
& K(x, x)=\lambda-1 \text { for all } x \in S^{n-1}, \\
& K(x, y)=-1 \text { if } x \cdot y \in[-1, t]\} .
\end{aligned}
$$


We safely write inf instead of min here because we do not know if the infimum is attained.

Proposition 10.1. Let $C \subseteq S^{n-1}$ be a subset of the unit sphere such that every pair of distinct points in $C$ has inner product lying in $[-1, t]$. Then its cardinality is at most $\bar{\vartheta}(G(n,[-1, t]))$.

Proof. Let $K$ be a kernel satisfying the conditions in (28). Then, by the positivity of the continuous kernel $K$ it follows that

$$
0 \leq \sum_{\left(c, c^{\prime}\right) \in C^{2}} K\left(c, c^{\prime}\right)=\sum_{c} K(c, c)+\sum_{c \neq c^{\prime}} K\left(c, c^{\prime}\right) \leq|C| K(c, c)-|C|(|C|-1),
$$

so that $|C|-1 \leq K(c, c)$ and we are done.

We finish by showing how the original formulation of the linear programming bound can be obtained from (28). Using Schoenberg's characterization (7) the semidefinite programming problem (28) simplifies to the linear programming problem

$$
\begin{aligned}
& \inf \left\{\lambda: f_{0} \geq 0, f_{1} \geq 0, \ldots, \sum_{k=0}^{\infty} f_{k} P_{k}^{(\alpha, \alpha)}(1)=\lambda-1\right. \\
& \left.\qquad \sum_{k=0}^{\infty} f_{k} P_{k}^{(\alpha, \alpha)}(u)=-1 \text { for all } u \in[-1, t]\right\} .
\end{aligned}
$$

We can strengthen it by requiring $\sum_{k=0}^{\infty} f_{k} P_{k}^{(\alpha, \alpha)}(u) \leq-1$ for all $u \in[-1, t]$. By restricting $f_{0}=0$ the infimum is not effected. Then, after simplification, we get the linear programming bound (cf. [DGS], [KL]).

$$
\inf \left\{1+\sum_{k=1}^{\infty} f_{k}: f_{1} \geq 0, f_{2} \geq 0, \ldots, \sum_{k=1}^{\infty} f_{k} P_{k}^{(\alpha, \alpha)}(u) \leq-1 \text { for all } u \in[-1, t]\right\} \text {. }
$$

By Proposition 10.1 it gives an upper bound for the maximal number of points on the unit sphere with minimal angular distance arccost.

Acknowledgements. We thank Dion Gijswijt, Gil Kalai, Tom Koornwinder, Pablo Parrilo, and Lex Schrijver for their helpful comments.

\section{References}

[AAR] G.E. Andrews, R. Askey, R. Roy, Special Functions, Cambridge University Press, 1999.

[B] C. BACHOC, Linear programming bounds for codes in Grassmannian spaces, IEEE Trans. Inf. Th. 52 (2006), 2111-2125.

[Bo] S. Bochner, Hilbert distances and positive definite functions, Ann. of Math. 42 (1941), 647-656.

[BrE] N.G. DE Bruijn, P. Erdös, A colour problem for infinite graphs and a problem in the theory of relations, Indagationes Math. 13 (1951), 369-373.

[D] P. Delsarte, An Algebraic Approach to the Association Schemes of Coding Theory, Philips Res. Rep. Suppl., 1973.

[DGS] P. Delsarte, J.M. Goethals, J.J. Seidel, Spherical codes and designs, Geom. Dedicata 6 (1977), 363-388. 
[F] K.J. FAlconer, The realization of distances in measurable subsets covering $\mathbb{R}^{n}, \mathrm{~J}$. Combin. Theory Ser. A 31 (1981), 187-189.

[FrR] P. Frankl, V. RÖDL, Forbidden intersections, Trans. Amer. Math. Soc. 300 (1987), 259-286.

[FrW] P. FrankL, R.M. Wilson, Intersection theorems with geometric consequences, Combinatorica 1 (1981), 357-368.

[KL] G.A. Kabatiansky, V.I. Levenshtein, Bounds for packings on a sphere and in space, Problems of Information Transmission 14 (1978), 1-17.

[Kn] D.E. Knuth, The Sandwich Theorem, Electron. J. Combin. 1 (1994).

[LR] D.G. Larman, C.A. Rogers, The realization of distances within sets in Euclidean space, Mathematika 19 (1972), 1-24.

[L1] L. Lovász, On the Shannon capacity of a graph, IEEE Trans. Inf. Th. 25 (1979), $1-7$.

[L2] L. LovÁsz, Self-dual polytopes and the chromatic number of distance graphs on the sphere, Acta Sci. Math. 45 (1983), 317-323.

[MRR] R.J. McEliece, E.R. Rodemich, H.C. Rumsey JR., The Lovász bound and some generalizations, J. Combin. Inf. System Sci. 3 (1978) 134-152.

[OV] F. De Oliveira Filho, F. Vallentin, Fourier analysis, linear programming, and densities of distance avoiding sets in $\mathbb{R}^{n}$, J. Eur. Math. Soc., to appear.

[R] A.M. Raigorodskit, On the chromatic number of a space, Uspekhi Mat. Nauk 55 (2000), 147-148.

[S] I.J. Schoenberg, Positive definite functions on spheres, Duke Math. J. 9 (1942), 96-108.

[Sc] A. SchriJver, A comparison of the Delsarte and Lovász bounds, IEEE Trans. Inf. Th. 25 (1979), 425-429.

[Sz] G. Szegö, Orthogonal Polynomials, Volume XXIII, Colloqium Publications, American Mathematical Society, 1967.

[Szé] L.A. SzÉKely, Erdős on unit distances and the Szemerédi-Trotter theorems, in "Paul Erdős and His Mathematics (G. Halász, L. Lovász, M. Simonovits, V.T. Sós, eds.), Springer, (2002), 649-666.

[SzéW] L.A. Székely, N.C. Wormald, Bounds on the measurable chromatic number of $\mathbb{R}^{n}$, Discrete Math. 75 (1989), 343-372.

[VK] N.JA. Vilenkin, A.U. KLIMYK, Representation of Lie Groups and Special Functions, Vol. 2, Kluwer Academic Publishers, 1993.

[W] G.N. Watson, A Treatise on the Theory of Bessel Function, Cambridge Mathematical Library, 1995.

Christine Bachoc, Laboratoire A2X, Université Bordeaux I, 351, cours de la Libération, 33405 Talence, France bachoc@math.u-bordeaux1.fr

Gabriele NeBE, Lehrstuhl D für Mathematik, RWTH Aachen University, Templergraben 64, 52062 Aachen, Germany nebe@math.rwth-aachen.de

Fernando Mário de Oliveira Filho, Centrum voor Wiskunde en Informatica (CWI), Kruislaan 413, 1098 SJ Amsterdam, The Netherlands f.m.de.oliveira.filho@cwi.nl Frank Vallentin, Delft Institute of Applied Mathematics, Technical University of Delft, P.O. Box 5031, 2600 GA Delft, The Netherlands f.vallentin@tudelft.nl

Received: April 1, 2008

Accepted: July 14, 2008

Open Access This article is distributed under the terms of the Creative Commons Attribution Noncommercial License which permits any noncommercial use, distribution, and reproduction in any medium, provided the original author(s) and source are credited. 